\title{
Implementing an Integrated Information System Designed for Romanian Public Entities
}

\author{
Meda UDROIU ${ }^{1}$, Mihail DUMITRACHE ${ }^{1}$, Ionuț SANDU**1, Adrian BREZULIANU² \\ ${ }^{1}$ National Institute for Research and Development in Informatics, 8-10 Maresal Averescu Avenue, \\ Bucharest, 01145, Romania \\ meda.udroiu@rotld.ro, mihail.dumitrache@ici.ro, ionut.sandu@ici.ro (*Corresponding author) \\ ${ }^{2}$ Greensoft SRL, 8 Arcu Avenue, "Voința" Building, 1-st floor, Iasi, 700137, Romania \\ adi.brezulianu@greensoft.com.ro
}

\begin{abstract}
This paper presents an integrated information system model designed to be used by the Romanian public entities. This system is part of a research project that promotes the public-private sector partnership in Romania. So, the main objective of the research presented in this paper is the development of an integrated information system for management of activities (IISMA) that provides the management of public institution information by making use of resources and activities, software functional modules and open-source computing applications. The solution proposed ensures the streamlining of the information flow, the improvement of the management of resources and inter-institutional inter-operability by offering a versatile, safe and resilient solution that can be customised depending on the specific requirements of the public entities. IISMA is based on open - source solutions and functional software specialization modules, aiming to reduce the complexity of the conceptual separation. IISMA ensures: functional disconnection of modules (keeping inter-operability), standardisation of functional modules, implementation of extendibility facilities and component re-use facilities and protection of data which is processed, used and stored.
\end{abstract}

Keywords: Integrated information system for management of activities, Interinstitutional inter-operability, Open-source solutions, NLP algorithms, GDPR platforms, IoT.

\section{Introduction}

Under the current technological developments, designing, analysing and implementing an integrated information system for the management of activities is a desideratum for any public or private institution. Analysing the literature one can detach diverse approaches and solutions, adopted by public institutions in Great Britain and Holland [26], and Spain [11]. Some recent studies [13,27] analyse the impact of cloud/ fog and big data technologies over integrated information systems for management of activities and also successful models implemented in Italy [5], Germany, Lithuania and Norway [10]. Studies highlight the effectiveness of implementing an integrated management system of activities. However, these studies are characterised by heterogeneous approaches over similar business processes that do not always use standards and methodologies recognised in the area and imply usage of diverse systems. This causes duplication of data and reduced opportunities for functional extensibility and integrability. The present proposal identifies modern architectures, based on services / micro-services [16] that facilitates GDPR compliance [5], increased security and resilience level in the field of information [3], and also inclusion of emergent technologies in the field of development of integrated computing systems for management of activities [9]. Among significant results one can mention implementation of services associated to critical infrastructures, with types such as "Identity", "Message Broker", "Business Processes", "Information Resources Registry". Potential beneficiaries of the integrated information system may be central and local public institutions, but also private entities interested in optimisation of operations peculiar to management of activities.

The remainder of this paper is organized as follows: Section 2 describes the general framework of the system followed by its functional and technical architecture. The main benefits of the system including several prerequisites for human-software interaction are briefly presented in Section 3. The final part of the paper summarizes the key features of the integrated system emphasizing the advantages of employing it by the Romanian public sector entities.

\section{Scientific and Technical Presentation of the System}

The main objective of this project is represented by the development of an Integrated Information System for Management of Activities (IISMA) that provides the management of records, resources and activities created and maintained by certain Romanian public institutions, by using software 


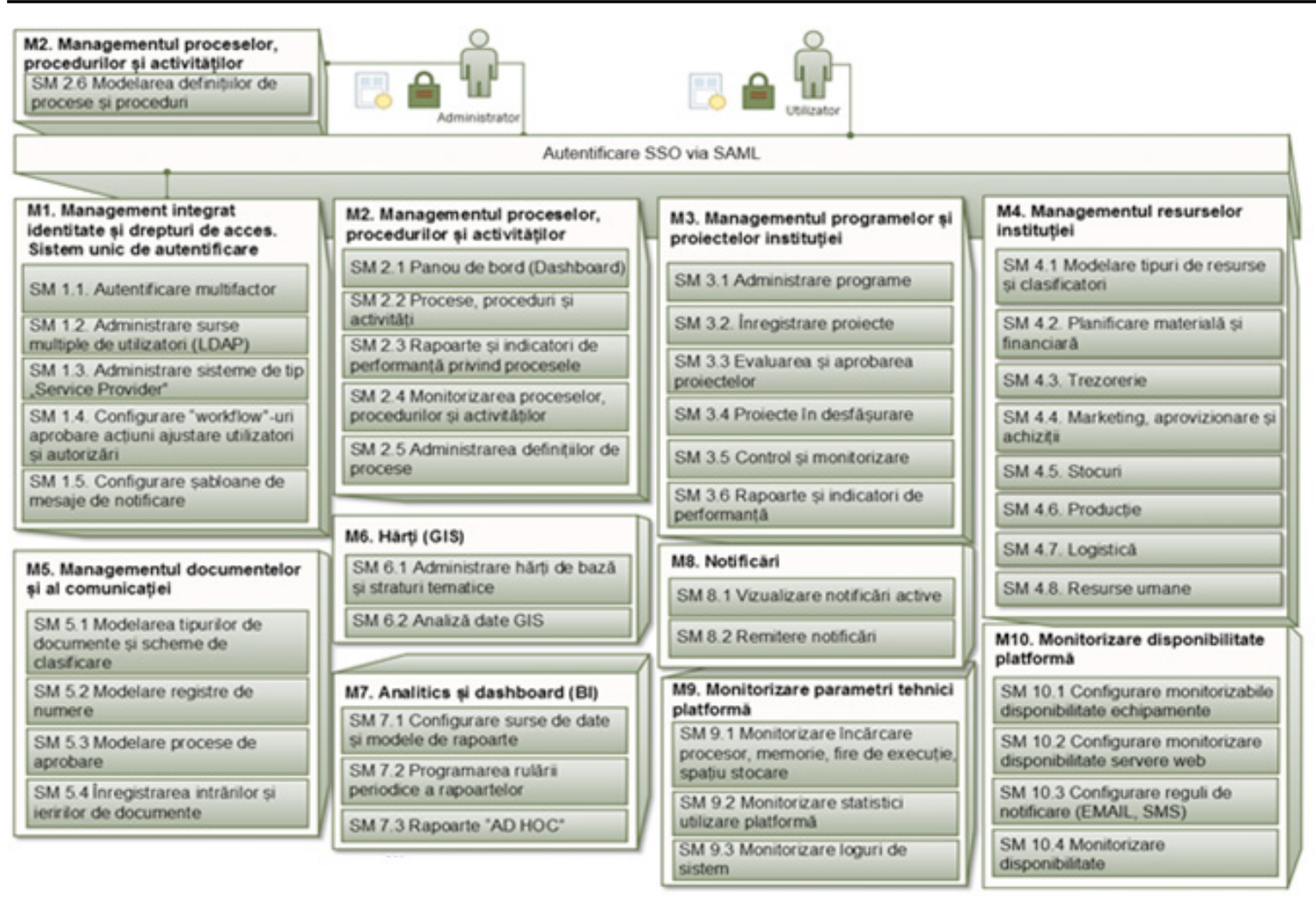

Figure 1. Functional architecture

functional modules and open-source computing applications. The solution proposed ensures the streamlining of the information flow, improvement of resources management, and inter-institutional inter-operability by offering a versatile, safe and resilient solution that can be customized depending on the specific requirements of the Romanian public entities. IISMA is based on open-source solutions and functional software specialization modules, aiming to reduce the complexity of the conceptual separation. IISMA ensures: functional disconnection of modules (keeping inter-operability), standardization of functional modules, implementation of extendibility facilities, component re-use facilities and securing of processed and stored data. In the process of designing the platform, the following are being pursued: functional disconnection through SOA architecture [24], standardization of components' interaction, standardization of principles of modelling, creating a layer of reusable infrastructure services at an organization level, a unique centralized identity service and generate premises of GDPR compliance [8] with regard to the processing of personal data, platform extensibility by creating connectors, securing the system at all its levels.

\subsection{Functional Architecture}

To achieve the objectives of the ToR and ensure the results, versatility and future development capability, the functional blocks of the platform are structured in 10 modules (Figure 1).

\section{M1. Module for integrated management} of identity and access rights. The unique authentication system ensures uniform administration of users ' accounts, authorization rules and offers, at the level of the platform and securing multi-factor authentication mechanisms by using the following functional blocks (BF):

- SM 1.1. Multi-factor type authentication implements the unique authentication system over the entire platform. For authentication, a layered identification mechanism is used, based on the combination of two or more factors such as the following:

- knowledge factors - information that only the user has (e.g. password, PIN)

- ownership factors - objects that only the user possesses (e.g. ATM cards, certificates) 
- inherent factors - user specific elements (e.g. digital fingerprint).

- SM 1.2. Multiple user sources administration configures one or more data sources (i.e. LDAP [17] or database), containing the data authentication. The authentication process takes into consideration all data sources.

- SM 1.3. "Service Provider" system administration allows inclusion of platform modules in an integrated authentication and authorization mechanism, and it can be extended to any other module that would be integrated within the platform later on.

- SM 1.4. Defining Work-flows for approval of adjustments facilitates configuration of workflows in several steps, automatic initiation of amendments at the moment of initiating account or authorization data modification, ensuring process controllability, traceability and safety.

- SM. 1.5. Configuring notification message templates used for events associated with user accounts modifications.

Innovation elements of this module are the following: inclusion of WSO2 Identity Server [39], SAML [23], x509 [29], OAUTH [19], multifactor authentication, SSL, modification control, security policies.

M2. Module for management of processes, procedures and activities offers functional capabilities for modelling and control over logical structures of processes, procedures and activities. This is structured in several submodules such as:

- SM 2.1. Dashboard synthesizes the status of ongoing processes, procedures and activities

- SM 2.2. Processes, procedures and activities lists the actions whose performance the current user is responsible for; to complete the action, the user completes the specific parameters according to the process definition and uploads support documents, digitally signed and validated.

- SM 2.3. Performance reports and indicators allow generating and exporting synthetic or detailed reports, highlighting the current situation or historical situations regarding processes and activities.

- $\quad$ SM 2.4. Monitoring of processes, procedures and activities facilitates consulting, in real time, through tables and graphics, the status of processes and also suspending or annulling actions.

- SM 2.5. Administration of definitions of processes, procedures and activities enables the recording, updating and deletion of process procedures and activities' definitions, expressed using the BPMN [4] or WS-BPEL [19] standards.

- SM 2.6. Modelling processes and procedures' definitions - represents an application desktop $[6,36]$ which offers the possibility of modelling graphs, using "drag and drop" in off-line mode, their definitions in the BPMN or WS-BPEL format. The resulting files are loaded onto the platform by SM 2.5.

The innovation elements of this module consists in the inclusion of WSO2 Business Process Server [35], BPMN [4], Activity [1], Apache Orchestration Director Engine (ODE) [2], WSBPEL [30], WSO2 Developer Studio [6,36], WSO2 Business Activity Monitor (BAM) [33], Apache Hadoop [2], MapReduce [34], SSL.

M3. Management of programs and projects of the institution with some modules such as:

- SM 3.1. Program administration ensures registering the programs along with run-time, goals / missions, priorities, indicators, budget structure, funds, support documentation and project proposal evaluation grids. The modification of programs is subject to simple multi-level approval using M2.

- SM 3.2. Project registration facilitates registration of project proposals at program level, correlating the objectives / missions, priorities, indicators and budget structure with those of the program. It enables the definition of groups of activities, periods of progress, the resources involved, the proposed results and deadlines. Also, it allows integration of several projects.

- SM 3.3. The evaluation and approval of projects allows to assess project proposals by filling out the evaluation grid, following the logic of evaluation process modelled via the M2. Approved project proposals become ongoing projects.

- SM 3.4. Ongoing projects provides easy access to the list of approved projects, with the possibility of consulting all the details and registration elements of the progress of 
projects (used, activities undertaken, outputs etc.). It allows uploading digitally signed documents. The adjustment of the project data is achieved through controlled processes according to M2.

- SM 3.5. Control and monitoring enables the analysis of the financial implementation and compliance with the deadlines for implementation. It allows the registration of control reports and updating the register of identified risks.

- SM 3.6. Performance reports and indicators are used to generate analytical / synthetic, tabular or graphical reports, at program / project level, on quantitative, qualitative and impact projections on program indicators.

The Innovation elements for this module are WSO2 Governance Registry [38], GeoServer [10], WMS [42], Leaflet [15], SSL.

\section{M4. Institution resource management with some modules such as:}

- SM 4.1. Modelling resource and ranker types allows modelling institution's resource types (products, fixed assets, assets, management, locations, partners, etc.) with their metadata as well as taxonomies for organizing the resources. Taxonomies may include departmental structure or other logical resource organization structure at department level.

- SM 4.2. Material and financial planning deals with planning, setting and monitoring budgets for revenue and expenditure, and procurement programs

- SM 4.3. The Treasury facilitates logical structuring and tracking of all transactions with monetary and other assets, financial and cash flows.

- $\quad$ SM 4.4. Marketing supplying and acquisitions allows registering purchase requisition, orders and purchasing processes. The buying process phases and evaluation of tenders is accomplished by module M2. It ensures estimations regarding the finalizing date and late notifications to acquisition staff, or its beneficiaries. Upon completion of the acquisition process, this module keeps records of the purchasing price for later reference.

- SM 4.5. The inventory gathers and stores information about the inventory movement, based on input / output documents approved through M2 specific processes.

- SM 4.6. The production facilitates management of recipes and means of production, operational and production costs, selling prices and input / consumption, automated generation of book keeping, production / consumption documents, and also obtaining specific reports, organizing and tracking production / selling / delivery activities and information on clients / beneficiaries, locations etc., and also processing the related documents.

- SM 4.7. Logistics enables registration of administrative and logistic support activities, planning of administrative, maintenance and support activities, and deployment of logistic resources (constructions / infrastructure elements, means of transportation etc.), recording information regarding their status and availability.

- SM 4.8. Human Resources allows the description of posts, the definition of job descriptions, staff specifications, the formation of organizational structures, the creation of the hierarchy, the distribution of the authority and the horizontal and vertical coordination of activities, as well as the differentiation of positions, group definition, organizational behaviour, organizational motivation, symbols, control and evaluation systems, management structure, formal structure and organization paradigm, management of processes / activities related to human resources (planning, attracting / recruiting, selecting and hiring staff or reducing the number of employees); management / performance (defining and measuring individual / group performance and identifying performance indicators and objectives, providing feedback on performance and correlating rewards / sanctions and / or promotions / disposals); career management and payroll; managing human resources development (learning opportunities, increasing skills and potential, training and improvement to improve individual, team and organizational performance, rotation of positions, organizing and conducting education, training and improving professional training)

The innovation elements involved in designing this module are: the inclusion of WSO2 Governance Registry [38], SSL. 
M5. Document and communication management with following modules

- SM 5.1. Modelling document types and classification schemes

- $\quad$ SM 5.2. Modelling number registries

- $\quad$ SM 5.3. Modelling approval processes

- SM 5.4. Recording of arrivals and departures of documents with the possibility of postscanning automatic loading and Basic code generation

Elements of innovation: the inclusion of WSO2 Governance Registry [38], SSL.

M6. Maps (GIS) with the following modules:

- SM 6.1. Map management - allows defining maps, the thematic layers and associated symbology, publication of WMS services.

- SM 6.2. GIS data analysis allows viewing geospatial map data, with the possibility of selecting thematic layers (e.g. buildings, terrains etc.), and creating geospatial perimeters of polygonal type. It can be integrated in any manner requiring geospatial representation of modelled resources.

Elements of innovation are the inclusion of GeoServer WMS [10] [42] [41], Shapefiles, Leaflet [15], SSL.

\section{M7. Analytics and dashboard (BI) with}

- SM 7.1. Configuration of data sources and report models.

- SM 7.2. Scheduling periodic running of reports allows to schedule their execution and transmission by e-mail to recipients you want to configure.

- SM 7.3. "AD HOC" Reports enables selection of the data source query, columns and data display rules.

Elements of innovation: the inclusion of WSO2 Analytics [32], WSO2 Business Activity Monitor [33], Report Server Community [22].

\section{M8. Notification with}

- SM 8.1. Visualize active notifications allows to view the list of current user notifications generated as a result of existing business process conditions, or manually generated notifications by another user.

SM 8.2. Submit notifications facilitates the definition of information structures of the notification and transmitting it to an addressee or a group of addressees within the system.

Elements of innovation for this module are the inclusion of WSO2 Enterprise Service Bus [56], WSO2 Message Broker [40]:

\section{M9. Monitoring platform technical parameters with}

- SM 9.1. Monitoring CPU load, memory, thread execution, storage space through graphical display tools

- $\quad$ SM 9.2. Usage statistics monitoring platform

- $\quad$ SM 9.3. Monitoring system logs

Elements of innovation: - the inclusion of WSO2 Carbon [36].

M10. Monitoring platform availability with:

- SM10.1. Configuration equipment availability monitoring which allows to define monitored equipment and software systems as well as the parameters being monitored.

- SM 10.2 Web servers availability monitoring configuration which enables the parameters of web servers to be monitored.

- SM 10.3. Configuration rules for notification (EMAIL, SMS) allows to define conditions to generate notifications at the overcoming of defined normality intervals.

- SM 10.4. Monitoring availability offers evaluation functions for technical status and availability of the platform by means of a graphical interface, which allows individual tracking of each component of the platform or component group level.

Elements of innovation are the inclusion of ZABBIX Server [28], Zabbix Agent, JMX [14], SNMP [25].

\subsection{Technical Architecture}

The technical architecture of the platform, is structured on three levels:

- DMZ Level ensures direct interfacing with the user and is represented by load 


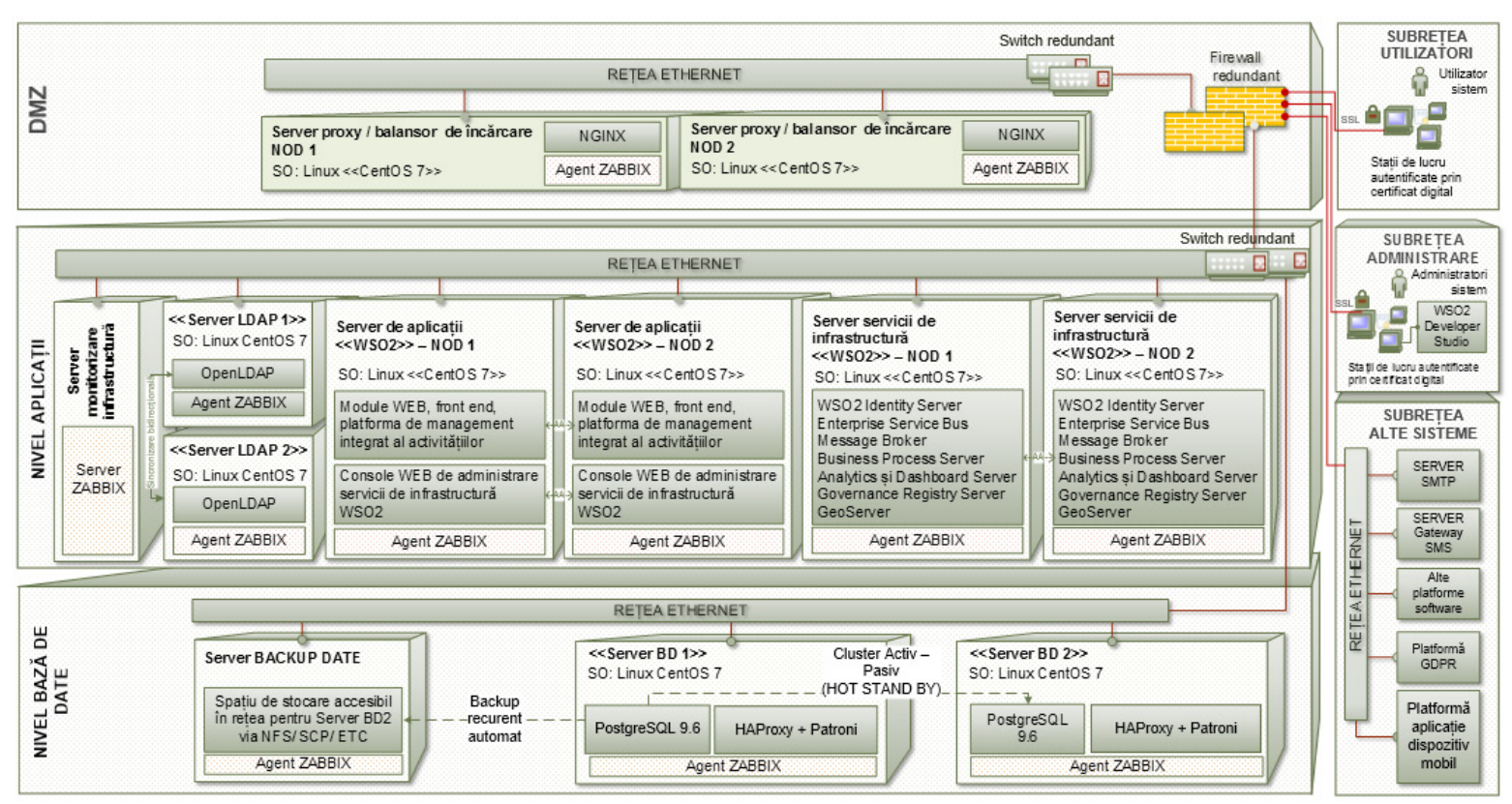

Figure 2. Technical architecture

rockers for balanced distribution of users' applications to viable application servers, i.e. viable infrastructure services, as well as moving traffic to the viable node in emergency situations. There are two load software rockers configurated in IP Failover mode to which the traffic will be re-directed from the firewall level one of them being used as a main rocker and the other one as a spare rocker.

The elements of innovation for this level are the inclusion of NGINX, SSL, Proxy/Reverse Proxy, authentication workstation with digital certificate.

- Level APPLICATION hosts clusters of applications servers, infrastructure services, LDAP servers, a server to monitor availability of the platform. The three clusters are presented in a two-node variant but can be extended depending on the assumed load level.

The elements of innovation in designing this level are the inclusion of WSO2 Application Server [31], OpenLDAP [15], WSO2 Enterprise Integrator [37], Enterprise Service Bus, WSSecurity [43], GeoServer [10], WSO2 Business Process Server [35].

- Level DATABASE will be provided by PostgreSQL 9.6 that will run in a cluster environment with multiple nodes, one of
- which is "master" and the rest are "hot stand-by nodes" to which data is replicated in real time.

The innovation consists in including PostgreSQL 9.6 [21], HAProxy [12], Patroni [20].

From the point of view of technological architecture, the platform is based on WSO2 technological pile.

The complexity degree of the platform is high due to the diversity of the types of functional blocks offered as well as to the multitude of technological components of the platform, as shown by the technical architecture, which makes it particularly necessary to group and specialize replicable functionalities in the form of centralized infrastructure services. The monitoring component, ZABBIX, plays an important role due to the ability to notify but also due to the possibility to perform some preventive actions with regard to some technical situations found.

\section{Discussion}

Currently, the system described above (IISMA) is designed to connect and interconnect ten Romanian public entities with their own characteristics into an integrated system that services all the entities' requests. The customer feedback has been positive which generated increased motivation of the research team members. 
Computing systems are not to be mistaken for computing platforms as the latter represent a complement of the information system by which the entire information system or a part of it can be conferred an electronic, virtual perspective for representation of resources, communication circuits of actors' identity, which will ensure by technological mechanisms, integrity and non-repudiation desiderata of the information resources, security, administrability, extensibility and availability. Moreover, within the modern information systems, mixing the responsibilities of the human actors, respectively of the virtual one (software system) is more and more striking, and this fusion needs a specific approach in terms related to the way the information systems have to be built in order to allow such types of interactions.

From a non-functional point of view, information systems at organization level, regardless of the functional spectre they serve, have to be able to comply, in a uniform manner, with the common norms, mechanisms and information circuits, which exist at the organization level and to be able to be connected to the information pipe of the organization. These desiderata regarding the software platforms which point rather to the essence of the problem than to its form can be achieved by meeting certain technical requirements during the design process related to their functional and technological structure.

At the basis of the assumptions considered in designing the system, the starting point is the observation and the theoretical classification of the elements with the stereotypical character, i.e. those with particular character that can be found in the information systems of the organization. Particularly, at the level of each organization or even subdivision of the organization, each of

\section{REFERENCES}

1. Activity BPMN, <https://www.activiti.org/>.

2. Apache Hadoop, <http://hadoop.apache.org/>.

3. Araújo, A., Melo, L., Andrade, L. H., Fonseca, J., Lima, J. N., Moreira, J., Maeda, R. \& Silva, T. The Strategy of Brazilian Government to Improve the Information Security Risk Management and the Cyber Security in Brazilian Public Sector. In The Third International Conference on Information Security and Digital Forensics (ISDF2017) 2017 Dec 8 (p. 27).

4. BPMN, <http://www.omg.org/spec/BPMN/2.0/> . the concepts such as the information resource, business process and identity, are customized according to the special characteristics of the information system. In this context, the specific elements of public entities are adequately designed by IISMA so that the interconnectivity and interoperability of all systems owned by Romanian public entities can be achieved.

\section{Conclusion}

An organisation's information system can be compared to the circulatory system of a living organism. It gathers all information resources that exist at a given moment within the organization, the circuits the information goes through within the organizational and trans-organizational framework, rules of access and usage. It also keeps track of all factors that interact in any way with the information resources, within the organization.

The model proposed in this paper highlights the functional objectives specific to an integrated information system. The system, together with its architecture, the instruments and the techniques it employs, turns into a platform that allows standardisation and customisation. Moreover, the platform creates the premises for extending the range of software instruments that are able to support and control processes within an organization.

\section{Acknowledgements}

This research work was supported by a grant on the Romanian Ministry of Innovation and Research, UEFISCDI, project number 8SOL/2018 within PNCDIII, project code:PNIII-P2-2.1-SOL-2017-09-0102, project name: Integrated Information System for Management of Activities (IISMA).
5. Broy, D. (2017). Germany: Starting Implementation of the GDPR-Brief Overview of the Government Bill for a New Federal Data Protection Act, Eur. Data Prot. L. Rev, 3(93).

6. Definirea grafică a proceselor de business de tip BPMN și WS-BPEL, <https://docs. wso2.com/display/BPS351/Creating+a+BPMN+Process+Using + Developer+Studio $>$.

7. Gagliardi, D., Schina, L., Sarcinella, M. L., Mangialardi, G., Niglia, F. \& Corallo, A. (2017 Jan 31). Information and communication technologies and public participation: 
interactive maps and value added for citizens, Government Information Quarterly, 34(1), 153-66.

8. GDPR, <http://data.consilium.europa.eu/doc/ document/ST-9565-2015-INIT/en/pdf>.

9. Gil-Garcia, J. R., Helbig, N. \& Ojo, A. (2014 Jun 30). Being smart: Emerging technologies and innovation in the public sector, Government Information Quarterly, 31(I), 1-8.

10. GeoServer, <http://geoserver.org/>.

11. Han, Y. \& Sun, R. (2016 Jul 1). Research on public management efficiency improvement method based on parallel database oriented optimization management information system, RISTI (Revista Iberica de Sistemas e Tecnologias de Informacao), (E5), 425-37.

12. HAProxy, <http://www.haproxy.org/>.

13. Jones, S., Irani, Z., Sivarajah, U. \& Love, P. E. (2017). Risks and rewards of cloud computing in the UK public sector: A reflection on three Organisational case studies, Information Systems Frontiers, 1-24.

14. JMX, <http://openjdk.java.net/groups/jmx/>.

15. Leaflet, $<$ http://leafletjs.com/index.html $>$.

16. Li, Y. \& Jiang, N. The design and implementation of multi-source application middleware based on service bus. In IOP Conference Series: Earth and Environmental Science 2017 Jun 69(1) (p. 012166). IOP Publishing.

17. LDAP, $<$ https://tools.ietf.org/rfc/rfc4511.txt $>$.

18. Mussari, R., Tranfaglia, A. E., Reichard, C., Bjørnå, H., Nakrošis, V. \& BankauskaitèGrigaliūnienè, S. (2016). Design, Trajectories of Reform, and Implementation of Performance Budgeting in Local Governments: A Comparative Study of Germany, Italy, Lithuania, and Norway, Local Public Sector Reforms in Times of Crisis, 101-119. Palgrave Macmillan UK.

19. OAUTH, <https://tools.ietf.org/html/rfc6749>.

20. Patroni, <https://github.com/zalando/patroni $>$.

21. Postgres - SGBD, <https://www.postgresql. org/docs/10/static/release-9-6.html $>$.

22. Report Server Community, <https:// reportserver.net/en/>.

23. SAML - Hughes et al. (March 2005). Profiles for the OASIS Security Assertion Markup Language (SAML) V2.0. OASIS Standard. Document identifier: saml-profiles-2.0-os, $<\mathrm{http}$ ://docs.oasis-open.org/security/saml/v2.0/ saml-profiles-2.0-os.pdf $>$.
24. SOA, <http://www.opengroup.org/standards/ soa $>$.

25. SNMP, $<$ https://tools.ietf.org/html/rfc1157>.

26. Van Dooren, W. \& Van de Walle, S. (editors). (2016 Apr 30). Performance information in the public sector: How it is used. Springer.

27. Zhang, Y., Qiu, M., Tsai, C. W., Hassan, M. M. \& Alamri, A. (2017 Mar). Health-CPS: Healthcare cyber-physical system assisted by cloud and big data, IEEE Systems Journal, 11(1), 88-95.

28. ZABBIX, <https://www.zabbix.com>.

29. x509, <https://tools.ietf.org/html/rfc5280>.

30. WS-BPEL, <http://docs.oasis-open.org/ wsbpel/2.0/wsbpel-v2.0.html>.

31. WSO2 Application Server, <https://wso2.com/ products/application-server/>.

32. WSO2 Analytics, $<$ https:// d o c s. w s o 2. co m/di s p la y/E I $611 /$ WSO2+Enterprise+Integrator+Analytics $>$.

33. WSO2 Business Activity Monitor, <https:// docs.wso2.com/display/BAM230/>.

34. WSO2 Business Activity Monitor MapReduce, $<$ https://docs.wso2.com/display/BAM240/Introduction+to+BAM+Analytics+Framework>.

35. WSO2 Business Process Server, $<$ https://docs. wso2.com/display/BPS360/>.

36. WSO2 Carbon, <https://wso2.com/products/ carbon/> [31] WSO2 Developer Studio $<$ https://wso2.com/products/developerstudio/>.

37. WSO2 Enterprise Integrator, <https://docs. wso2.com/display/EI611>.

38. WSO2 Governance Registry, https://wso2. $\mathrm{com} /$ products/governance-registry/.

39. WSO2 Identity Server, <https://wso2.com/ identity-and-access-management>.

40. WSO2 Message Broker, <https://docs.wso2. com/display/MB320/>.

41. ShapeFile, < <https://www.esri.com/library/ whitepapers/pdfs/shapefile.pdf $>$.

42. WMS - Web Map Service, <http://docs. geoserver.org/stable/en/user/services/wms/ index.html>.

43. WS - SECURITY, <https://www.oasisopen.org/committees/tc_home.php?wg abbrev $=$ wss $>$.

44. WSO2 Enterprise Service Bus, <https://wso2. com/products/enterprise-service-bus/ $>$. 